\title{
Variation in Primary Cesarean Delivery Rates by Individual Physician within a Single Hospital Laborist Model
}

\author{
Torri D. METZ, MD, MS ${ }^{1,2}$, Ms. Amanda A. ALLSHOUSE, MS ${ }^{3}$, Sara A Babcock GILBERT, \\ MD $^{1}$, Ms. Reina DOYLE, MPH ${ }^{2}$, Ms. Angie TONG, BS ${ }^{4}$, and J. Christopher CAREY, MD ${ }^{1,2}$ \\ 1 \\ 2 \\ 3 \\ 4
}

\section{Abstract}

Background-Laborist practice models are associated with lower cesarean delivery rates than individual private practice models in several studies; however, this effect is not uniform. Further exploration of laborist models may help us better understand the observed reduction in cesarean delivery rates in some hospitals with implementation of a laborist model.

Objective-Our objective was to evaluate the degree of variation in primary cesarean delivery rates by individual laborists within a single institution employing a laborist model. In addition, we sought to evaluate whether differences in cesarean delivery rates resulted in different maternal or short-term neonatal outcomes.

Study Design-At this teaching institution, one laborist (either a generalist or maternal-fetal medicine attending physician) is directly responsible for labor and delivery management during each shift. No patients are followed in a private practice model nor are physicians incentivized to perform deliveries. We retrospectively identified all laborists who delivered nulliparous, term women with cephalic singletons at this institution from 2007-14. Overall and individual primary cesarean delivery rates were reported as percentages with exact Pearson 95\% CI. Laborists were grouped by tertile as having low, medium or high cesarean delivery rates. Characteristics of the women delivered, indications for cesarean delivery, and short-term neonatal outcomes were compared between these groups. A binomial regression model of cesarean delivery was estimated, where the relative rates of each laborist compared to the lowest-unadjusted laborist rate were calculated; a second model was estimated to adjust for patient-level maternal characteristics.

Correspondence: Torri D. Metz, MD, MS, Denver Health Medical Center, Department of Obstetrics and Gynecology, 777 Bannock St, MC 0660, Denver, CO 80204, Torri.Metz@dhha.org, Work: 303-602-9727, Cell: 303-656-5477, Fax: 303-602-9734 Word count: Abstract 455, Main text 3203.

Publisher's Disclaimer: This is a PDF file of an unedited manuscript that has been accepted for publication. As a service to our customers we are providing this early version of the manuscript. The manuscript will undergo copyediting, typesetting, and review of the resulting proof before it is published in its final citable form. Please note that during the production process errors may be discovered which could affect the content, and all legal disclaimers that apply to the journal pertain.

The authors report no conflict of interest.

Accepted for oral presentation at the Society for Maternal-Fetal Medicine $36^{\text {th }}$ Annual Meeting, Atlanta, GA, February 1-6, 2015. 
Results-Twenty laborists delivered 2,224 nulliparous, term women with cephalic singletons. The overall cesarean delivery rate was $24.1 \%$ (95\% CI 21.4-26.8). In an unadjusted binomial model, the overall effect of individual laborist was significant ( $\mathrm{p}<0.001)$, and a 2.9 fold $(1.5,5.4$, $\mathrm{p}=0.001$ ) variation between the cesarean delivery rates of the highest (35.9\%) and lowest $(12.5 \%)$ physicians was observed. When adjusted for hypertensive disease, gestational age at delivery, race, and maternal age, the physician effect remained overall significant $(\mathrm{p}=0.0265)$ with the difference between physicians expanding to 3.58 (1.72-7.47, $\mathrm{p}<0.001)$. Between groups of laborists with low, medium, and high cesarean delivery rates, patient demographics and clinical characteristics of the population managed were clinically similar and not different statistically. The primary indication for cesarean delivery did not differ between groups. Similarly there were no differences in short-term neonatal outcomes including Apgar scores, arterial cord blood $\mathrm{pH}$ or incidence of neonatal encephalopathy.

Conclusion-The 3-fold variation in cesarean delivery rates between laborists at the same institution without observed differences in patient characteristics or short-term neonatal outcomes draws attention to the impact of individual physician decision-making on cesarean delivery rates even within a laborist care model. Further exploration of the role of individual physician decisionmaking on cesarean rates may help to better elucidate the effect of the laborist model.

\section{Keywords}

cesarean; cesarean delivery rate; laborist; physician traits

\section{Introduction}

With the ongoing rise in the rate of cesarean delivery in the United States, many organizations and investigators are working to develop guidelines and evidence-based practice to help stabilize and ultimately reduce the number of cesarean deliveries. Prevention of the primary cesarean delivery has gained traction as the most obvious point of intervention to prevent future cesarean deliveries and future morbidity associated with cesarean deliveries. The American College of Obstetricians and Gynecologists (ACOG) and the Society for Maternal-Fetal Medicine developed a consensus statement with guidelines to help prevent cesarean deliveries which included suggestions for tolerated length of second stage, use of operative vaginal delivery in appropriate candidates, and length of augmentation prior to performing a cesarean delivery for arrest disorders. ${ }^{1}$

Other investigators have evaluated different care models and a "laborist" (or obstetric hospitalist) model has been found to reduce the rate of cesarean delivery when compared to traditional models of obstetrical care. ${ }^{2-4}$ An ACOG Committee Opinion defines a laborist as an obstetrician-gynecologist who is employed by the hospital to manage laboring patients and attend to obstetric emergencies. ${ }^{5}$ Given this definition, this reduction in cesarean delivery is plausible with the increased availability of the laborist, focused expertise in labor management, and potentially a greater objectivity in assessing the clinical scenario when not managing a private patient. However, there is a large study in which the same reduction in cesarean delivery was not observed with a laborist model. ${ }^{6}$ In addition a survey-based study noted an association between laborists and an increased likelihood to perform cesarean delivery when provided with clinical vignettes. ${ }^{7}$ 
It may be that the observed reduction in cesarean delivery with the laborist model in some studies is a reflection of not just the laborist care model, but also the individual laborists. Prior training, experience, comfort with specific skills, and cognitive traits likely all influence care of individual physicians in a given clinical scenario. These differences between individuals may result in differing rates of primary cesarean delivery even within the same clinical setting.

Our objective was to evaluate the degree of variation in primary cesarean delivery rates by individual laborists within a single institution employing a laborist model to better understand the observed reduction in cesarean delivery observed in some studies.

\section{Materials and Methods}

This was a retrospective cohort study of all women who delivered at a single center from December 2007 through May 2014. Women who delivered over this time period were identified from the electronic medical record charting system used on Labor and Delivery for nursing charting (OBTraceVue). Nulliparous women with a term, cephalic singleton pregnancy and no contraindication to vaginal delivery (previa, active herpes infection, HIV with a viral load $>1000$ copies $/ \mathrm{mL}$, previous uterine surgery, malpresentation) were included. All women who were managed primarily by our midwifery service were excluded from this study. This study was approved by the Colorado Multiple Institutional Review Board.

Data for the cohort were collected from three sources: OBTraceVue, individual patient medical records, and the Denver Health Data Warehouse. The Denver Health Data Warehouse is an institution-wide administrative database of all admissions and out-patient visits within the Denver Health system. The Data Warehouse contains both demographic information and clinical data including billing codes and all laboratory results for each admission. The Data Warehouse was utilized to extract demographic characteristics (payor source, race and ethnicity), cord blood gases, and billing codes for hypertensive disease (preeclampsia, chronic hypertension and gestational hypertension), pre-existing diabetes and gestational diabetes.

Clinical obstetric data were primarily extracted from nursing charting in OBTraceVue with supplemental detailed chart abstraction by two obstetricians (TDM and SABG) for intrapartum course and indication for primary cesarean delivery. When the primary indication for cesarean delivery was not clear in the operative report, we developed an algorithm for assigning the primary and secondary indication for cesarean delivery. In cases in which it was unclear whether the primary indication was an arrest disorder or nonreassuring fetal status, the arrest disorder was selected as the primary indication and nonreassuring fetal status was selected as the secondary indication. Analyses were then performed to assess the primary indication, secondary indication, and non-reassuring fetal status as either the primary or secondary indication to evaluate if there were differences between physician group by indication for cesarean delivery. 
All deliveries in the cohort were assigned to the laborist (attending physician) who staffed the delivery. All laborists in the practice were included in the analysis regardless of the number of deliveries managed. Laborists at this institution are assigned to shifts on Labor and Delivery months in advance to allow for in-house laborist coverage at all times. No patients in the practice are followed in a private practice model. Resident physicians are involved in the care of all women on Labor and Delivery. The laborist on-call is responsible for the intrapartum management of all women in labor and any obstetric emergencies. We do not perform elective inductions at our institution, and women are not scheduled for inductions or scheduled procedures on a day when a particular laborist is staffing the floor. This differs from a university coverage model in that the laborists are assigned only to Labor and Delivery. There are not competing out-patient responsibilities, and none of the women seen in our practice identify a particular attending as their private care provider. Laborist coverage assignments are based on the clinical time of individual physicians and are distributed as a mix of daytime and night and weekend coverage. Within our laborist model, education of residents and back-up of midwives and family practice physicians is also provided consistent with the definition of a laborist by ACOG. ${ }^{5}$

The primary cesarean delivery rate for each attending laborist was calculated by dividing the number of cesarean deliveries for each laborist by the total number of deliveries staffed by that individual laborist over the study time period. Individual laborist cesarean delivery rates are reported with exact Pearson 95\% confidence intervals (CI).

Individual laborists were then divided into three groups by tertile of low, medium and high cesarean delivery rates. To determine if significant differences existed in the population of women managed by the three groups of laborists, the basic demographics and clinical characteristics of the women they delivered were compared. Short-term neonatal outcome data were compared across provider tertile to determine if different rates of cesarean delivery were associated with differences in outcomes. Comparisons between groups were made using chi square for categorical and ANOVA for continuous measures.

A binomial regression model of cesarean delivery was estimated using SAS Glimmix, where the relative rates of each laborist compared to the lowest-unadjusted laborist rate were calculated; a second model was estimated to adjust for patient-level maternal characteristics (hypertensive disease, race, maternal age, gestational age at delivery) associated with cesarean delivery to further assess the effect of individual laborists on cesarean delivery.

Individual physician characteristics (male versus female, years in practice, maternal-fetal medicine versus generalist, and delivery volume) were also evaluated to determine if there was an association between these factors and cesarean delivery rates. Comparisons were made by physician tertile and modeled using a general linear mixed effects model to adjust for within-physician correlation.

$\mathrm{P}<0.05$ was considered statistically significant. All analyses were completed in SAS 9.4, with graphics created using Graphpad Prism v6.03. Data from clinical chart abstraction were managed prior to analysis using the secure, web-based application Research Electronic Data Capture (REDcap) hosted at University of Colorado. ${ }^{8}$ 


\section{Results}

The total number of women who delivered at our institution over the 7-year study time period was 22,006. There were 4139 term, nulliparous deliveries in cephalic presentation with an overall rate of cesarean delivery of $18 \%$ among these women. After excluding deliveries managed by our midwifery service $(n=1658)$ and deliveries without a documented laborist as the attending $(\mathrm{n}=257)$, the overall cesarean delivery rate of our analyzed cohort was $24.1 \%$ (95\% CI 22.4-26.0).

Twenty laborists delivered the 2,224 nulliparous, term women with cephalic singletons included in our analysis. The number of deliveries per laborist ranged from 11 to 390 (interquartile range, 52-139).

In the tertile with the lowest cesarean delivery rate, 7 laborists had a total of 559 deliveries between them; with individual rates of CS between $12.5 \%$ and $22.1 \%$. The 6 laborists in the tertile with the medium cesarean delivery rate had 951 deliveries between them; with rates of cesarean ranging from 23.1 to 24.6. In the tertile with the highest cesarean delivery rate, 7 laborists had 714 deliveries between them; with rates of cesarean delivery ranging from 25.0 to $35.9 \%$. We assessed the internal validity of grouping the laborists into tertiles. In accordance with the defined tertiles, there were statistically significant differences between groups for cesarean delivery rate (19\% low, $24 \%$ medium, $29 \%$ high, $\mathrm{p}<0.001)$.

In an unadjusted binomial model, the overall effect of individual laborist was significant $(\mathrm{p}<0.001)$, and a 2.9 fold $(1.5,5.4, \mathrm{p}=0.001)$ variation between the cesarean delivery rates of the highest (35.9\%) and lowest (12.5\%) physicians was observed (Figure 1). When adjusted for hypertensive disease, gestational age at delivery $(<39 \mathrm{w}, 39 \mathrm{w}, 40 \mathrm{w}, 41 \mathrm{w}+)$, race (black, hispanic, white, other), maternal age $(<18$ years, 19-<25, 25-<30, 30-<35, and 35+), and the hypertension and gestational age interaction, the physician effect remained overall significant $(\mathrm{p}=0.0265)$ with the difference between physicians expanding to 3.58 (1.72-7.47, $\mathrm{p}<0.001)$. Between groups of laborists with low, medium, and high cesarean delivery rates, there were no significant differences in patient demographics or clinical characteristics of the population managed (Table 1).

Among only the women who had a cesarean delivery, the percentage of women with an arrest disorder as the primary indication for cesarean delivery did not differ between groups. Similarly the percentage of women with non-reassuring fetal status as the primary indication for cesarean delivery did not differ between groups (Table 2). However, as expected, the percentage of all women in the cohort (cesarean delivery/total population managed) who underwent cesarean delivery increased across tertiles for both indications $(14.7 \%, 16.0 \%$, $20.0 \%$ for arrest disorders and 4.5\%, 7.0\%, 8.5\% for non-reassuring fetal status). Nonreassuring fetal status was the primary or secondary indication for cesarean delivery in $5.9 \%$ versus $7.9 \%$ versus $10.2 \%$ of women managed. In short, laborists in the highest tertile did more cesarean deliveries for both of these indications.

Detailed data abstraction of the electronic medical records of women who underwent primary cesarean delivery in the cohort allowed us to compare other clinical characteristics of the women managed by the three groups of laborists with low, medium and high cesarean 
rates. There was no statistically significant difference in maternal outcomes including length of second stage of labor for women who reached the second stage, time at maximum cervical dilation prior to cesarean delivery, incidence of chorioamnionitis, hysterectomy or need for blood transfusion (Table 2).

When operative vaginal delivery (OVD) utilization was compared pairwise between laborists with high cesarean delivery rates versus the other laborists in the cohort combined, significantly lower rates of operative vaginal delivery were found in the high cesarean delivery tertile when compared to the other laborists ( $4.9 \%$ versus $7.7 \%, \mathrm{p}=0.015)$. The overall difference across the three laborist groups in operative vaginal delivery rate was also significant $(\mathrm{p}=0.01)$, however the pattern of operative vaginal delivery rate and cesarean delivery tertile was not monotonic (Table 3), as the medium laborist tertile had the highest rate of operative vaginal delivery.

Short-term neonatal outcomes were compared across laborist tertile, and no differences were found between groups in percentage of neonates with Apgar scores less than 7 at 5 minutes $(3.07 \%, 3.61 \%, 3.70 \%, \mathrm{p}=0.83)$, or incidence of neonatal encephalopathy $(0.18 \%, 0 \%, 0 \%$, $\mathrm{p}=0.25$ ). There was also not a statistically significant correlation between arterial cord blood $\mathrm{pH}$ and cesarean delivery rate (Figure 2).

There were 9 deliveries with an arterial cord blood pH less than 7.0 (range 6.88-6.97). These cases were reviewed in detail. 6 of these cases were delivered by laborists in the low cesarean rate group, 3 in the medium cesarean rate group, and none in the high cesarean rate group. In all of these cases, there were no apparent long-term sequelae of the fetal acidemia. For the one case of neonatal encephalopathy in the cohort, the arterial cord pH was 7.06 with a base excess of -15 . This neonate was delivered by a laborist in the low cesarean rate group. In this case, the patient was in spontaneous labor at 40 weeks and 5 days. She was ultimately delivered by cesarean at $5-6 \mathrm{~cm}$ dilation for arrest of dilation. Intermittent variable decelerations and two episodes of fetal bradycardia which resolved with oxygen and position changes were noted prior to delivery. The cesarean delivery was not performed for non-reassuring fetal status. The neonate had a normal MRI and no seizure activity. However, he was discharged with a clinical diagnosis of neonatal encephalopathy.

We also evaluated individual laborist characteristics that may influence cesarean delivery rates. Among the 20 laborists, there was not a statistically significant correlation between cesarean delivery rate and overall delivery volume (Pearson correlation $0.01,-0.43$ to 0.45 , $\mathrm{p}=0.9)$. Years in obstetric practice since graduation from residency, maternal-fetal medicine specialist versus general obstetrician-gynecologist and male versus female was also modeled, adjusting for within-physician correlation in a binomial regression model, with cesarean delivery as the outcome. None of these individual physician characteristics were predictive of cesarean delivery in the model, nor were there significant differences when these physician characteristics were compared across the tertiles of low, medium and high cesarean rates with a chi square test. Detailed data are not presented to preserve the anonymity of the individual laborists.

Am J Obstet Gynecol. Author manuscript; available in PMC 2017 April 01. 


\section{Comment}

We found a 3-fold variation in cesarean delivery rates between laborists at the same institution. We did not find evidence of differing maternal clinical characteristics, indications for cesarean delivery, nor short-term neonatal outcomes between laborist groups with low, medium and high cesarean delivery rates. These findings draw attention to the impact of individual physician decision-making on cesarean delivery rates even within a laborist care model.

At our institution a collaborative laborist model has been in place for many years and likely contributes to our overall lower primary cesarean delivery rate of $18.0 \%$ (including midwifery deliveries) for nulliparous women at term over the study time period compared to $28 \%$ in the United States in $2009 .{ }^{9}$ Comparisons of the clinical characteristics of women delivered by the different groups of laborists with low, medium and high cesarean delivery rates demonstrates the essentially random allocation of patients to individual providers by a pre-defined schedule made months in advance. Even within a laborist model in which the laborists manage a clinically similar patient population, there was large variation in cesarean delivery rates by individual laborists. There was not a difference in indication for cesarean delivery between the groups indicating that some individuals may be less likely to perform a cesarean delivery for any indication. This is consistent with previously published literature. ${ }^{10}$

Physician traits have been only minimally explored as a factor that influences the rise in cesarean delivery rates in the United States. Yee and colleagues demonstrated that lower physician anxiety levels and more proactive coping were associated with higher rates of trial of labor after cesarean and successful vaginal birth after cesarean when managing women with a prior cesarean delivery. ${ }^{11}$ Further exploration of how specific physician traits affect outcomes is warranted. Ultimately interventions to assist with modification of behavior in clinical scenarios may help to reduce the cesarean delivery rate and influence patient outcomes.

Interventions such as circulating individual physician cesarean delivery rates and implementation of protocols have resulted in reduction of cesarean delivery rates. ${ }^{12}$ More recent publications have focused on differences in outcomes with laborist compared to traditional private practice models. Several of these publications have demonstrated benefits of laborist models ${ }^{2-4}$, while others fail to demonstrate a difference in outcomes, and specifically cesarean delivery rates, with and without laborists. ${ }^{6}$ Based on our findings, some of this heterogeneity of results may depend on the individual laborists hired.

While there were not differences in our short-term neonatal outcome measures of 5-minute Apgar scores or incidence of neonatal encephalopathy among the groups, there were more cases of arterial $\mathrm{pH}$ less than 7.0 in the low cesarean rate group (6 of the 9 cases were managed by laborists in the low cesarean rate group). In several of the cases, delivery was expedited following a catastrophic intrapartum event necessitating emergent cesarean delivery or operative vaginal delivery. However, the higher number of cases with an arterial cord $\mathrm{pH}$ less than 7.0 may also be indicative of a higher threshold for performing cesarean 
delivery which in rare cases results in adverse outcomes. Further study is needed to help determine the "optimal" rate of cesarean delivery for nulliparous women at term to maximize vaginal deliveries while still maintaining optimal maternal and fetal outcomes.

\section{Strengths and Limitations}

Our study is limited by relying on retrospective chart abstraction for indication for cesarean delivery. In addition, our study is based on a single center and may not be generalizable to other patient populations or hospitals. However, the uniformity in setting and clinical characteristics of the patients managed at this single institution allowed us to more effectively compare cesarean delivery rates on a more level "playing field" without extraneous factors that may otherwise affect decisions to proceed with cesarean delivery. This is also, therefore, a strength of the study.

The study is also limited by assigning the delivery to the laborist who was present at the time of delivery. We recognize that this may not be the laborist who made the decisions that led to the ultimate outcome (cesarean versus vaginal birth). We assessed a surrogate marker (delivery within 1 hour of shift change) to evaluate if the low cesarean group essentially deferred deliveries by cesarean to the next shift. There was not difference by tertile in the number of cesarean deliveries performed within an hour of shift change (data not shown). While not a perfect measure, the mode of delivery decision is generally made by the delivering physician, and we felt this was a reasonable approach for the purposes of this analysis.

We also excluded births to women whose labors were managed by our midwifery service. Our midwives typically manage low-risk, unmedicated births, and it did not seem appropriate to assign the cesarean deliveries of midwife patients to the delivering attending when we would be unable to assign the vaginal births of patients delivered by midwives to a specific laborist as they practice independently and do not consult the laborist service for the majority of their births. However, even when we included the cesarean deliveries for midwife-managed patients in the numerator for the cesarean delivery rate, we still observed similar variations in cesarean delivery rates by laborist, and laborists essentially fell into the same tertiles as assigned when the midwife births were excluded. We therefore do not suspect that exclusion of the midwife births significantly impacted our conclusions.

\section{Conclusions}

The success of laborist models is likely dependent on both the care model itself and individual laborist decision-making which has the potential to dramatically affect overall cesarean delivery rates. Reduction of the rate of cesarean delivery in the United States is complex and will require multiple targeted approaches to be successful. Further exploration of whether physician cognitive traits impact clinical outcomes and whether these traits can be modified to improve patient outcomes is needed. 


\section{Acknowledgment}

The authors would like to thank Josh Durfee, Statistical Research Specialist, and Renee Starr, RN Informatics Specialist, at Denver Health Medical Center for their assistance with the data extraction for this manuscript.

Financial support: This work was supported by the Agency for Healthcare Research and Quality grant number R24 HS022143-01, and National Institutes of Health/National Center for Research Resources Colorado CTSI grant number UL1 TR001082.

\section{References}

1. American College of Obstetricians and Gynecologists, Society for Maternal-Fetal Medicine. et al. Safe prevention of the primary cesarean delivery. American journal of obstetrics and gynecology. 2014; 210(3):179-193. [PubMed: 24565430]

2. Iriye BK, Huang WH, Condon J, et al. Implementation of a laborist program and evaluation of the effect upon cesarean delivery. American journal of obstetrics and gynecology. 2013; 209(3):251, e251-256. [PubMed: 23904102]

3. Nijagal MA, Kuppermann M, Nakagawa S, Cheng Y. Two practice models in one labor and delivery unit: association with cesarean delivery rates. American journal of obstetrics and gynecology. 2015; 212(4):491, e491-498. [PubMed: 25446697]

4. Rosenstein MG, Nijagal M, Nakagawa S, Gregorich SE, Kuppermann M. The Association of Expanded Access to a Collaborative Midwifery and Laborist Model With Cesarean Delivery Rates. Obstet. Gynecol. 2015; 126(4):716-723. [PubMed: 26348175]

5. Committee opinion no. 459: the obstetric-gynecologic hospitalist. Obstet. Gynecol. 2010; 116(1): 237-239. [PubMed: 20567197]

6. Feldman DS, Bollman DL, Fridman M, et al. Do laborists improve delivery outcomes for laboring women in California community hospitals? American journal of obstetrics and gynecology. 2015; 213(4):587, e581-587, e513. [PubMed: 26026921]

7. Cheng YW, Snowden JM, Handler S, Tager IB, Hubbard A, Caughey AB. Clinicians' practice environment is associated with a higher likelihood of recommending cesarean deliveries. J. Matern. Fetal Neonatal Med. 2014; 27(12):1220-1227. [PubMed: 24224916]

8. Harris PA, Taylor R, Thielke R, Payne J, Gonzalez N, Conde JG. Research electronic data capture (REDCap)--a metadata-driven methodology and workflow process for providing translational research informatics support. Journal of biomedical informatics. 2009; 42(2):377-381. [PubMed: 18929686]

9. Osterman MJ, Martin JA. Trends in low-risk cesarean delivery in the United States, 1990-2013. Natl. Vital Stat. Rep. 2014; 63(6):1-16.

10. Li T, Rhoads GG, Smulian J, Demissie K, Wartenberg D, Kruse L. Physician cesarean delivery rates and risk-adjusted perinatal outcomes. Obstet. Gynecol. 2003; 101(6):1204-1212. [PubMed: 12798526]

11. Yee LM, Liu LY, Grobman WA. Relationship between obstetricians' cognitive and affective traits and delivery outcomes among women with a prior cesarean. American journal of obstetrics and gynecology. 2015; 213(3):413, e411-417. [PubMed: 25981847]

12. Socol ML, Garcia PM, Peaceman AM, Dooley SL. Reducing cesarean births at a primarily private university hospital. American journal of obstetrics and gynecology. 1993; 168(6 Pt 1):1748-1754. discussion 1754-1748. [PubMed: 8317517] 


\section{Rate of Cesarean Delivery by Individual Laborist}

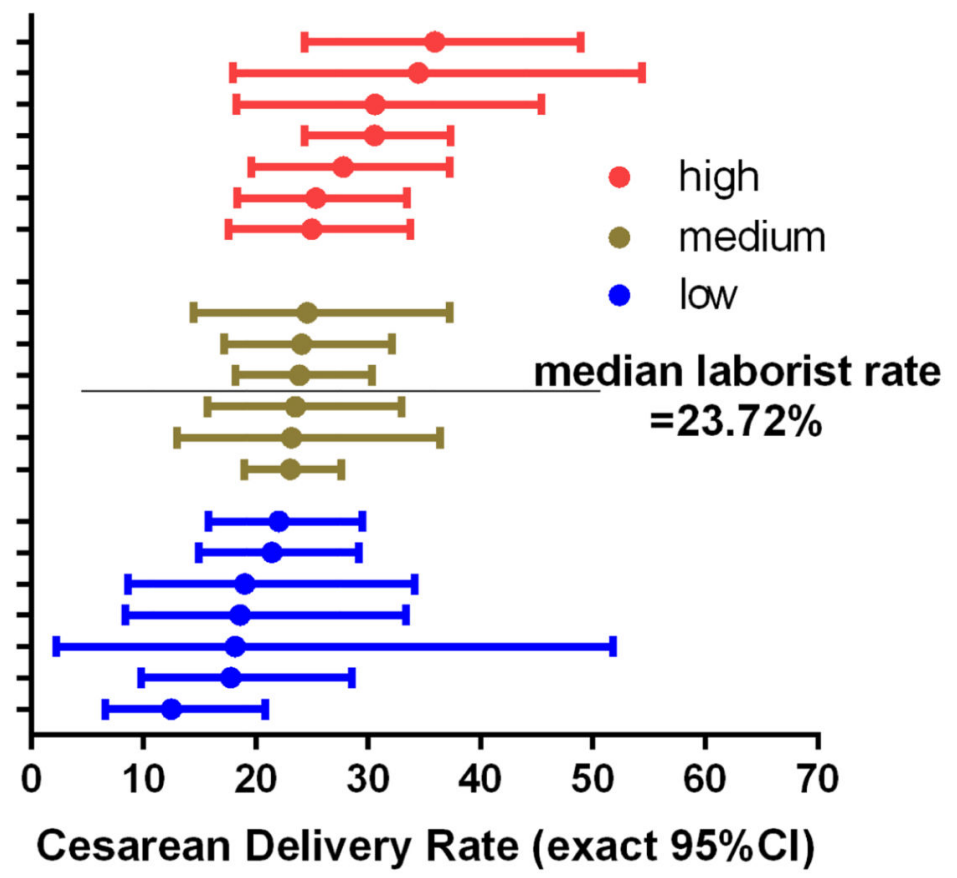

Figure 1.

Graphical representation of rates of cesarean delivery by individual laborist. Rates are reported with exact Pearson 95\% confidence intervals. The median rate of cesarean delivery was $23.7 \%$. Laborists were grouped into low, medium and high rates of cesarean delivery by tertile. 


\section{Relationship Between Arterial Cord Blood pH and Cesarean Delivery Rate}

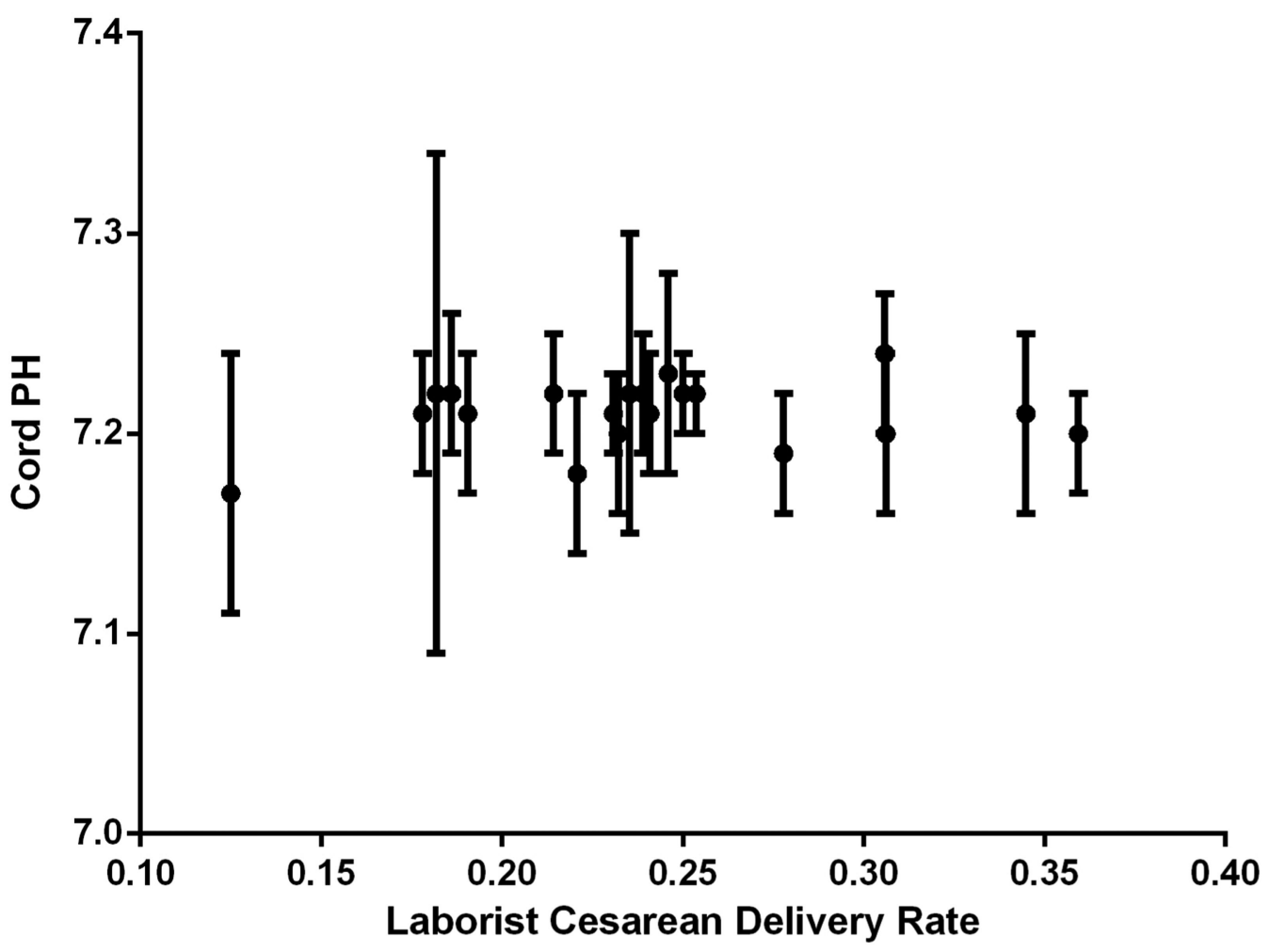

Figure 2.

There was not a statistically significant correlation between arterial cord blood $\mathrm{pH}$ and cesarean delivery rates (Pearson correlation $0.15,-0.32$ to $0.55, \mathrm{p}=0.54$ ). In the figure, individual laborists are represented by the vertical bars with ascending cesarean delivery rates on the $\mathrm{x}$-axis and arterial cord blood $\mathrm{pH}$ on the $\mathrm{y}$-axis. Only a subset of women had a cord blood gas performed ( $n=141$ low, $n=170$ medium, $n=184$ high) for clinical purposes that could be analyzed here. 
Table 1

Patient Characteristics by Groups of Physicians with Low, Medium and High Primary Cesarean Delivery Rates

\begin{tabular}{|c|c|c|c|c|}
\hline CHARACTERISTICS & $\underset{(n=559)}{\text { Low Cesarean Rate }}$ & $\underset{(n=951)}{\text { Medium Cesarean Rate }}$ & $\begin{array}{c}\text { High Cesarean Rate } \\
(n=714)\end{array}$ & p-value \\
\hline Race & & & & 0.77 \\
\hline White & $88(15.94)$ & $149(16.00)$ & $121(17.24)$ & \\
\hline Hispanic & $353(63.95)$ & $625(67.13)$ & $452(64.39)$ & \\
\hline Black & $77(13.95)$ & $110(11.82)$ & $91(12.96)$ & \\
\hline Other & $34(6.16)$ & $47(5.05)$ & $38(5.41)$ & \\
\hline Maternal age, geomean (95\% CI) & $22.6(22.2,23.0)$ & $22.3(22.0,22.7)$ & $22.6(22.2,23.0)$ & 0.53 \\
\hline Induction & $134(25.2)$ & $215(23.5)$ & $180(25.9)$ & 0.50 \\
\hline Cervical dilation at admission, mean (SE) & $3.56(0.10)$ & $3.57(0.09)$ & $3.41(0.10)$ & 0.45 \\
\hline Gestational age at delivery, mean (SE) & $39.7(0.05)$ & $39.7(0.04)$ & $39.6(0.05)$ & 0.89 \\
\hline Hypertensive disease $^{a}$ & $123(22.0)$ & $208(21.9)$ & $179(25.1)$ & 0.26 \\
\hline Gestational or preexisting diabetes & $51(9.1)$ & $73(7.7)$ & $45(6.3)$ & 0.17 \\
\hline
\end{tabular}

All values are $\mathrm{n}(\%)$ unless otherwise indicated.

${ }^{a}$ Hypertensive disease includes women with chronic hypertension, gestational hypertension, or preeclampsia. 
Table 2

Clinical Characteristics of Women Undergoing Primary Cesarean Delivery by Groups of Physicians with Low, Medium and High Cesarean Delivery Rates

\begin{tabular}{|c|c|c|c|c|}
\hline CHARACTERISTIC & $\begin{array}{l}\text { Low Cesarean } \\
\text { Rate }(\mathbf{n}=\mathbf{1 0 7})\end{array}$ & $\begin{array}{l}\text { Medium Cesarean } \\
\text { Rate }(n=224)\end{array}$ & $\begin{array}{l}\text { High Cesarean } \\
\text { Rate }(n=206)\end{array}$ & p-value \\
\hline $\begin{array}{l}\text { Primary indication for cesarean of non-reassuring fetal } \\
\text { status }\end{array}$ & $25(23.4)$ & $67(29.9)$ & $61(29.6)$ & 0.437 \\
\hline Primary indication for cesarean of arrest disorder & $82(76.6)$ & $152(67.9)$ & $143(69.4)$ & 0.257 \\
\hline $\begin{array}{l}\text { Primary or secondary indication for cesarean of non- } \\
\text { reassuring fetal status }\end{array}$ & $33(30.84)$ & $75(33.5)$ & $73(35.4)$ & 0.727 \\
\hline Chorioamnionitis & $26(24.3)$ & $70(31.3)$ & $44(21.5)$ & 0.061 \\
\hline $\begin{array}{l}\text { Hours at maximum dilation prior to cesarean (hrs), } \\
\text { mean (SE) }\end{array}$ & $6.35(0.59)$ & $6.85(0.61)$ & $5.64(0.38)$ & 0.223 \\
\hline Length of $2^{\text {nd }}$ stage (hrs) ${ }^{a}$, mean (SE) & $4.01(0.28)$ & $4.76(0.27)$ & $4.35(0.29)$ & 0.243 \\
\hline$\geq 3$ hours of 2 nd stage ${ }^{a}$ & $19(17.8)$ & $38(17.0)$ & 39 (18.9) & 0.864 \\
\hline Transfusion & $0(0.00)$ & $5(2.23)$ & $4(1.94)$ & 0.380 \\
\hline Hysterectomy & $0(0.00)$ & $1(0.45)$ & $0(0.00)$ & $>0.99$ \\
\hline
\end{tabular}

All values are n (\%) unless otherwise indicated.

Only women who had a cesarean delivery are included in this table.

${ }^{a}$ Only n=121 women were completely dilated at the time of cesarean delivery. The length of $2^{\text {nd }}$ stage data were collected only for women who reached complete dilation prior to cesarean delivery. 
Table 3

Mode of Delivery Among Vaginal Births by Groups of Physicians with Low, Medium and High Cesarean Delivery Rates

\begin{tabular}{|l|c|c|c|c|}
\hline MODE OF DELIVERY & Low Cesarean Rate (n=559) & Medium Cesarean Rate (n=951) & High Cesarean Rate (n=714) & p-value \\
\hline Forceps or vacuum & $35(6.3)$ & $81(8.5)$ & $35(4.9)$ & 0.013 \\
Forceps & $22(3.9)$ & $61(6.4)$ & $18(2.5)$ & $<0.001$ \\
Vacuum & $13(2.3)$ & $20(2.1)$ & $17(2.4)$ & 0.933 \\
\hline
\end{tabular}

All values are n (\%). 